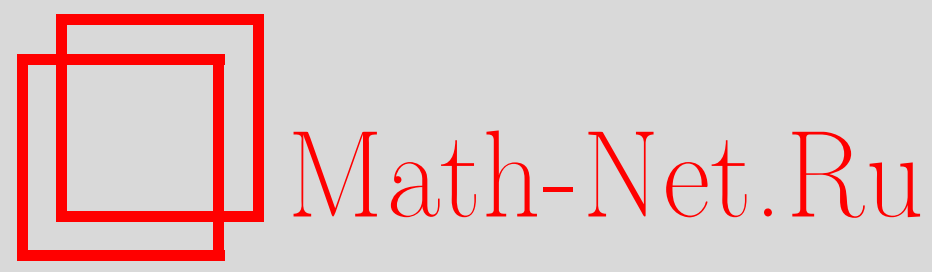

Г. И. Ивченко, М. В. Соболева, Некоторые неравновероятные модели случайных подстановок, Дискрет. матем., 2011, том 23, выпуск 3, 23-31

DOI: https://doi.org/10.4213/dm1150

Использование Общероссийского математического портала Math-Net.Ru подразумевает, что вы прочитали и согласны с пользовательским соглашением http://www . mathnet.ru/rus/agreement

Параметры загрузки:

IP: 54.162 .85 .209

26 апреля 2023 г., $14: 17: 43$ 
УДК 519.24

\title{
Некоторые неравновероятные модели случайных подстановок
}

\author{
() 2011 г. Г. И. Ивченко, М. В. Соболева
}

Рассматривается двухпараметрическая модель случайных $n$-подстановок, обобщающая классическую модель $A$-подстановок, для которой изучается совместное распределение числа $A$-циклов и $\bar{A}$-циклов при различных конкретизациях подмножества $A \subset X_{n}=\{1,2, \ldots, n\}$

\section{1. Введение}

В $[1,2]$ была введена общая параметрическая модель случайных подстановок, согласно которой произвольная подстановка $s$ из симметрической группы $S_{n}$ подстановок $n$-й степени наблюдается с вероятностью, пропорциональной $\prod_{i} \theta_{i}^{c_{i}}$, где $c_{i}-$ число циклов длины $i$ подстановки $s, i=1,2, \ldots, n, \sum_{i} i c_{i}=n$ и $\theta=\left(\theta_{1} \ldots, \theta_{n}\right), \theta$ - параметр меры $\mathbf{P}_{\theta}$ :

$$
\mathbf{P}_{\theta}(s)=I\left(\sum_{i=1}^{n} i c_{i}=n\right) \frac{1}{H_{n}(\theta)} \prod_{i=1}^{n} \theta_{i}^{c_{i}} .
$$

Здесь $I(\cdot)$ - индикатор и $H_{n}(\theta)$ - необходимый нормирующий множитель, имеющий вид

$$
H_{n}(\theta)=n !\left[z^{n}\right] \exp \left\{\sum_{i=1}^{n} \frac{z^{i} \theta_{i}}{i}\right\},
$$

здесь и далее $\left[z^{n}\right] f(z)=\operatorname{coef}_{z^{n}} f(z)$ - коэффициент при $z^{n}$ в $f(z)$.

Пусть $\bar{c}(n)=\left(c_{1}, \ldots, c_{n}\right)$ есть цикловая структура случайной подстановки в модели (1) и

$$
F_{n, \theta}(t)=\mathbf{E}_{\theta} \prod_{i=1}^{n} t_{i}^{c_{i}}
$$

- ее производящая функция. Тогда $[1,2]$

$$
F_{n, \theta}(t)=\frac{H_{n}(t \times \theta)}{H_{n}(\theta)},
$$

где $t=\left(t_{1}, \ldots, t_{n}\right)$ и $t \times \theta=\left(t_{1} \theta_{1}, \ldots, t_{n} \theta_{n}\right)$.

Как отмечается в [2], соотношение (3) может быть основой для изучения различных структурных свойств подстановок в рамках общей модели (1). В [2] также приводится ряд 
конкретных примеров применения такого общего подхода к изучению случайных подстановок, но при этом отмечается, что более детальное исследование случайных подстановок в общей модели (1) ждет своего решения.

В свете изложенного в [2], представляется естественным для продвижения в этой тематике рассмотреть различные конкретизации общей модели $P_{\theta}$ (скажем, ограничившись лишь двухпараметрическими моделями) и при этом акцентировать внимание на изучении лишь важнейших структурных характеристик подстановки (типа общего числа циклов конкретного типа). Такой характер и имеет настоящая работа. Мы рассматриваем некоторые естественные двухпараметрические модели, обобщающие классические (то есть равновероятные) модели $A$-подстановок, и в рамках этих моделей изучаем совместное распределение чисел $A$-циклов и $\bar{A}$-циклов случайной подстановки при различных конкретизациях множества $A \subseteq X_{n}=\{1,2, \ldots, n\}$.

\section{2. Постановка задачи и предварительные соотношения}

Рассматривается множество $S_{n}=\{s\}$ подстановок степени $n$ ( $n$-подстановок), то есть множество из $n$ ! взаимно однозначных отображений множества $X_{n}=\{1,2, \ldots, n\}$ в себя. Каждая подстановка, как известно, может быть разложена в произведение независимых циклов. Говорят, что $n$-подстановка $s$ принадлежит цикловому классу $\left\{1^{c_{1}}, 2^{c_{2}}, \ldots, n^{c_{n}}\right\}$, если она имеет $c_{i}$ циклов длины $i, i=1,2, \ldots, n$. Набор $\tilde{c}(n)=\left(c_{1}, c_{2}, \ldots, c_{n}\right)$ называется цикловой структурой подстановки $s$ (ясно, что всегда $\left.\sum_{i=1}^{n} i c_{i}=n\right)$.

Пусть $A$ есть заданное подмножество множества $X_{n}$ и $\bar{A}=X_{n} \backslash A$. Циклы подстановки $s$, длины которых являются элементами подмножества $A$ (соответственно, подмножества $\bar{A})$ будем называть $A$-циклами (соответственно, $\bar{A}$-циклами). Обозначим $c_{A}(n)\left(c_{\bar{A}}(n)\right)$ общее число $A$-циклов ( $\bar{A}$-циклов) подстановки $s$ :

$$
c_{A}(n)=\sum_{i \in A} c_{i}, \quad c_{\bar{A}}(n)=\sum_{i \in \bar{A}} c_{i},
$$

и введем на множестве $S_{n}$ вероятностную меру, приписывающую каждой подстановке $s \in S_{n}$ вес, пропорциональный $\theta_{1}^{c_{A}(n)} \theta_{2}^{c_{\bar{A}}(n)}$, где $\theta=\left(\theta_{1}, \theta_{2}\right), \theta_{1}, \theta_{2} \geqslant 0$, - параметры меры. Эта мера представляет собой специальный случай общей меры (1) и имеет вид

$$
\mathbf{P}_{\theta}(s)=I\left(\sum_{i=1}^{n} i c_{i}=n\right) \frac{\theta_{1}^{c_{a}(n)} \theta_{2}^{c_{\bar{A}}(n)}}{H_{n A}(\theta)}
$$

где

$$
H_{n A}(\theta)=n !\left[z^{n}\right] \exp \left\{\theta_{1} \sum_{i \in A} \frac{z^{i}}{i}+\theta_{2} \sum_{i \in \bar{A}} \frac{z^{i}}{i}\right\} .
$$

Здесь выражение в показателе экспоненты может быть заменено на

$$
\left(\theta_{1}-\theta_{2}\right) \sum_{i \in A} \frac{z^{i}}{i}+\theta_{2} \sum_{i=1}^{\infty} \frac{z^{i}}{i}=-\theta_{2} \ln (1-z)+\left(\theta_{1}-\theta_{2}\right) \sum_{i \in A} \frac{z^{i}}{i},
$$

либо на

$$
\theta_{1} \sum_{i=1}^{\infty} \frac{z^{i}}{i}\left(\theta_{2}-\theta_{1}\right) \sum_{i \in \bar{A}} \frac{z^{i}}{i}=-\theta_{1} \ln (1-z)+\left(\theta_{2}-\theta_{1}\right) \sum_{i \in \bar{A}} \frac{z^{i}}{i},
$$


следовательно,

$$
\begin{aligned}
H_{n A}(\theta) & =n !\left[z^{n}\right](1-z)^{-\theta_{2}} \exp \left\{\left(\theta_{1}-\theta_{2}\right) \sum_{i \in A} \frac{z^{i}}{i}\right\} \\
& =n !\left[z^{n}\right](1-z)^{-\theta_{1}} \exp \left\{\left(\theta_{2}-\theta_{1}\right) \sum_{i \in \bar{A}} \frac{z^{i}}{i}\right\} .
\end{aligned}
$$

Далее, для производящей функции пары $\left(c_{A}(n), c_{\bar{A}}(n)\right)$ из представления (3) следует равенство

$$
\Phi_{n \theta}\left(t_{1}, t_{2} ; A\right)=\mathbf{E}_{\theta} t_{1}^{c_{A}(n)} t_{2}^{c_{\bar{A}}^{(n)}}=\frac{H_{n A}\left(t_{1} \theta_{1}, t_{2} \theta_{2}\right)}{H_{n A}(\theta)} .
$$

Нашей задачей является исследование распределения двумерной характеристики $\left(c_{A}(n), c_{\bar{A}}(n)\right)$ случайной подстановки в рассматриваемой (двухпараметрической) модели для различных вариантов заданий подмножества $A \subseteq X_{n}$.

Но прежде мы выделим некоторые, представляющее и самостоятельный интерес, следствия соотношений (6) и (7).

1. Положив $t_{1}=t_{2}=t$ в (7), получаем производящую функцию важнейшей характеристики случайной подстановки - общего числа ее циклов

$$
c(n)=c_{A}(n)+c_{\bar{A}}(n)=\sum_{i=1}^{n} c_{i}
$$

именно,

$$
\mathbf{E}_{\theta} t^{c(n)}=\frac{H_{n A}\left(t \theta_{1}, t \theta_{2}\right)}{H_{n A}(\theta)} .
$$

Если, кроме того, $\theta_{1}=\theta_{2}=\theta>0$, то мы приходим к известной однопараметрической модели Эвенса [4], когда каждая подстановка $s \in S_{n}$ наблюдается с вероятностью, пропорциональной $\theta^{c(n)}$. Для этого случая представление (6) принимает вид

$$
\begin{aligned}
H_{n A}(\theta) & =n !\left[z^{n}\right](1-z)^{-\theta}=(-1)^{n} n !\left(\begin{array}{c}
-\theta \\
n
\end{array}\right) \\
& =\theta(\theta+1) \cdots(\theta+n-1)=[\theta]_{n}
\end{aligned}
$$

(эту комбинаторную формулу в дальнейшем мы будем неоднократно использовать), и мы приходим к известному результату [3]:

$$
\mathbf{E}_{\theta} t^{c(n)}=\frac{[t \theta]_{n}}{[\theta]_{n}}
$$

Этот случай (распределение числа циклов $c(n)$ в модели Эвенса) детально исследован в литературе (см. [3]), в то же время представление (7) для двумерной случайной величины $\left(c_{A}(n), c_{\bar{A}}(n)\right)$, принимающее в данном случае вид

$$
\Phi_{n, \theta}\left(t_{1}, t_{2} ; A\right)=\frac{1}{[\theta]_{n}} n !\left[z^{n}\right](1-z)^{-\theta t_{2}} \exp \left\{\theta\left(t_{1}-t_{2}\right) \sum_{i \in A} \frac{z^{i}}{i}\right\},
$$

является новым результатом и для модели Эвенса. 
2. Если в модели (5) положить $\theta_{2}=0, \theta_{1}=\theta>0$, то получим меру, сосредоточенную на подмножестве подстановок, имеющих лишь $A$-циклы, такие подстановки называются $A$-подстановками [5] и их изучению в рамках классической (равновероятной) модели посвящена обширная литература (см. [6, 7] и библиографию в них). Предложенная здесь модель позволяет, таким образом, изучать и неравновероятные $A$-подстановки.

Пусть $S_{n}(A)$ обозначает подмножество $A$-подстановок в $S_{n}$. Если на этом подмножестве задана параметрическая мера

$$
\mathbf{P}_{\theta}(s)=\frac{1}{H_{n A}(\theta)} I\left(\sum_{i \in A} i a_{i}=n\right) \theta^{c_{A}(n)}, \quad s \in S_{n}(A),
$$

где теперь

$$
H_{n A}(\theta)=n !\left[z^{n}\right] \exp \left\{\theta \sum_{i \in A} \frac{z^{i}}{i}\right\},
$$

то производящая функция общего числа циклов $c_{A}(n)$ такой случайной $A$-подстановки имеет вид

$$
\Phi_{n \theta}(t ; A)=\mathbf{E}_{\theta} t^{c_{A}(n)}=\frac{H_{n A}(t \theta)}{H_{n A}(\theta)},
$$

где $H_{n A}(\theta)$ дано в (10).

Если здесь положить $\theta=1$, то мы получаем равновероятную на $S_{n}(A)$ модель: каждая подстановка $s \in S_{n}(A)$ наблюдается с вероятностью $H_{n A}^{-1}(1)$.

Замечание 1. Случай $\theta_{1}=0, \theta_{2}=\theta>0$ соответствует симметричному варианту $\bar{A}$-подстановкам.

Далее мы разберем ряд наиболее популярных в литературе классов $A$-подстановок в контексте изложенного подхода.

3. Отметим, что из представления (7) следует общая формула для смешанных факториальных моментов случайных величин $c_{A}(n)$ и $c_{\bar{A}}(n)$ :

$$
\begin{aligned}
\mathbf{E}_{\theta}\left(c_{A}(n)\right)_{r_{1}}\left(c_{\bar{A}}(n)\right)_{r_{2}} & =\left.\frac{\partial^{r_{1}+r_{2}}}{\partial t_{1}^{r_{1}} \partial t_{2}^{r_{2}}} \Phi_{n, \theta}\left(t_{1}, t_{2} ; A\right)\right|_{t_{1}=t_{2}=1} \\
& =\theta_{1}^{r_{1}} \theta_{2}^{r_{2}} \frac{\partial^{r_{1}+r_{2}}}{\partial \theta_{1}^{r_{1}} \partial \theta_{2}^{r_{3}}} H_{n A}\left(\theta_{1} \theta_{2}\right) / H_{n A}(\theta),
\end{aligned}
$$

здесь и далее

$$
(a)_{r}=a(a-1) \cdots(a-r+1), \quad r \geqslant 1, \quad(a)_{0}=1 .
$$

Введя для краткости обозначение

$$
f_{\theta}(z)=\exp \left\{\theta_{1} \sum_{i \in A} \frac{z^{i}}{i}+\theta_{2} \sum_{i \in \bar{A}} \frac{z^{i}}{i}\right\},
$$


отсюда, в частности, получаем следующие представления для первых двух моментов:

$$
\begin{aligned}
\mathbf{E}_{\theta} c_{A}(n) & =\theta_{1}\left[z^{n}\right]\left(\sum_{i \in A} \frac{z^{i}}{i}\right) f_{\theta}(z) /\left[z^{n}\right] f_{\theta}(z), \\
\mathbf{E}_{\theta} c_{\bar{A}}(n) & =\theta_{2}\left[z^{n}\right]\left(\sum_{i \in \bar{A}} \frac{z^{i}}{i}\right) f_{\theta}(z) /\left[z^{n}\right] f_{\theta}(z), \\
\mathbf{E}_{\theta} c_{A}^{2}(n) & =\theta_{1}^{2}\left[z^{n}\right]\left(\sum_{i \in A} \frac{z^{i}}{i}\right)^{2} f_{\theta}(z) /\left[z^{n}\right] f_{\theta}(z)+\mathbf{E}_{\theta} c_{A}(n), \\
\mathbf{E}_{\theta} c_{\bar{A}}^{2}(n) & =\theta_{2}^{2}\left[z^{n}\right]\left(\sum_{i \in \bar{A}} \frac{z^{i}}{i}\right)^{2} f_{\theta}(z) /\left[z^{n}\right] f_{\theta}(z)+\mathbf{E}_{\theta} c_{\bar{A}}(n), \\
\mathbf{E}_{\theta} c_{A}(n) c_{\bar{A}}(n) & =\theta_{1} \theta_{2}\left[z^{n}\right]\left(\sum_{i \in A} \frac{z^{i}}{i}\right)\left(\sum_{i \in \bar{A}} \frac{z^{i}}{i}\right) f_{\theta}(z) /\left[z^{n}\right] f_{\theta}(z),
\end{aligned}
$$

Эти общие представления могут быть основой для вычисления моментов рассматриваемых характеристик случайной подстановки при различных конкретизациях подмножества $A$.

\section{3. $d$-инволюции}

В теории подстановок важное значение имеют решения уравнения

$$
s^{d}=e, \quad s \in S_{n},
$$

где $d-$ натуральное число, а $e-$ единичная подстановка, эти решения мы будем называть $d$-инволюциями. Известно, что такие подстановки состоят из циклов, длины которых являются делителями числа $d$. Таким образом, решения уравнения (13) образуют подмножество $A$-подстановок $S_{n}(A)$ при $A=\{i: i \mid d\}, d \geqslant 2, i \mid d$ означает, что $i$ делит $d$.

Если $d=2$, то решение уравнения (13) называется просто инволюцией. Инволюция содержит только циклы длин 1 и 2 и, следовательно, является $A$-подстановкой при $A=\{1,2\}$.

Будем считать, что $d-$ простое число, тогда функция $H_{n A}(\theta$ (см. (6)) для этого случая имеет вид

$$
\left.H_{n A}(\theta)=n !\left[z^{n}\right](1-z)^{-\theta} \exp \left\{\theta_{1}-\theta_{2}\right)\left(z+z^{d} / d\right)\right\} .
$$

Разложим здесь экспоненту в ряд по степеням $z$ и получим

$$
\begin{aligned}
\left.\exp \left\{\theta_{1}-\theta_{2}\right)\left(z+z^{d} / d\right)\right\} & =\left(\sum_{r \geqslant 0} \frac{\left(\theta_{1}-\theta_{2}\right)^{r}}{r !} z^{r}\right)\left(\sum_{j \geqslant 0} \frac{\left(\theta_{1}-\theta_{2}\right)^{j}}{d^{j} j !} z^{d j}\right) \\
& =\sum_{m \geqslant 0} a_{m, d}\left(\theta_{1}-\theta_{2}\right) z^{m},
\end{aligned}
$$


где

$$
a_{m, d}(u)=\sum_{j \leqslant m / d} \frac{u^{m-(d-1) j}}{d^{j} j !(m-d j) !},
$$

и запишем явное представление для $H_{n A}(\theta)$ в виде комбинаторной суммы

$$
H_{n A}(\theta)=n ! \sum_{m=0}^{n} \frac{a_{m, d}\left(\theta_{1}-\theta_{2}\right)}{(n-m) !}\left[\theta_{2}\right]_{n-m}
$$

Производящая функция (11) числа циклов в такой случайной $A$-подстановке имеет, следовательно, вид

$$
\Phi_{n \theta}(t ; A)=\frac{a_{n, d}(t \theta)}{a_{n, d}(\theta)} \frac{\sum_{j \leqslant n / d} \frac{(t \theta)^{n-(d-1) j}}{d^{j} j !(n-j d) !}}{\sum_{j \leqslant n / d} \frac{\theta^{n-(d-1) j}}{d^{j} j !(n-j d) !}} .
$$

\section{4. $d$-конгруэнтность}

Будем говорить, что подстановка $s \in S_{n}$ обладает свойством $d$-конгруэнтности, если длины всех циклов кратны числу $d \geqslant 2$, то есть эта подстановка есть $A$-подстановка при $A=\{k d, k=1,2, \ldots\}$.

При таком задании множества $A$ формула (6) принимает вид

$$
\begin{aligned}
H_{n A}(\theta) & =n !\left[z^{n}\right](1-z)^{-\theta_{2}} \exp \left\{\left(\theta_{1}-\theta_{2}\right) \frac{1}{d} \sum_{k \geqslant 1} \frac{z^{k d}}{k}\right\} \\
& =n !\left[z^{n}\right](1-z)^{-\theta_{2}} \exp \left\{-\frac{\left(\theta_{1}-\theta_{2}\right)}{d} \ln \left(1-z^{d}\right)\right\} \\
& =n !\left[z^{n}\right](1-z)^{-\theta_{2}}\left(1-z^{d}\right)^{-\left(\theta_{1}-\theta_{2}\right) / d},
\end{aligned}
$$

что можно записать в виде следующей комбинаторной суммы:

$$
H_{n A}(\theta)=\sum_{k \leqslant n / d} \frac{\left[\left(\theta_{1}-\theta_{2}\right) / d\right]_{k}\left[\theta_{2}\right]_{n-k d}}{k !(n-k d) !} .
$$

Отсюда мы можем получить явные формулы как для совместной производящей функции (7) пары $\left(c_{A}(n), c_{\bar{A}}(n)\right)$, где в данном случае $c_{A}(n)-$ число $d$-конгруэнтных циклов случайной подстановки, а $c_{\bar{A}}(n)-$ число остальных (не конгруэнтных) ее циклов, так и для производящей функции (12) числа циклов случайной $A$-подстановки. В последнем случае (при $\theta_{2}=0, \theta_{1}=\theta>0$ ) формула (19) принимает вид

$$
H_{n A}(\theta)=\frac{n !}{(n / d) !}[\theta / d]_{n / d},
$$

если $n$ кратно $d$, и $H_{n A}(\theta)=0$ в противном случае. 
Отметим важный частный случай $d=2$. В этом случае $c_{A}(n)\left(c_{\bar{A}}(n)\right)-$ число циклов четной (нечетной) длины в подстановке, и для подстановок четной степени, то есть при $n=2 n_{0}$, из (12) и (20) следует, что

$$
\begin{aligned}
\Phi_{2 n_{0} \theta}(t ; A) & =\mathbf{E}_{\theta} t^{c_{A}(n)}=[t \theta / 2]_{n_{0}} /[\theta / 2]_{n_{0}} \\
& =t \frac{(t \theta+2)(t \theta+4) \cdots\left(t \theta+2\left(n_{0}-1\right)\right)}{(\theta+2)(\theta+4) \cdots\left(\theta+2\left(n_{0}-1\right)\right)} \\
& =t \prod_{i=1}^{n_{0}-1}\left(q_{\theta i}+t p_{\theta i}\right),
\end{aligned}
$$

где

$$
p_{\theta i}=1-q_{\theta i}=\frac{\theta}{\theta+2 i}
$$

Из представления (21) следует, что $\Phi_{2 n_{0}, \theta}(t ; A)$ есть производящая функция независимых бернуллиевских случайных величин, таким образом, в данном случае имеет место представление

$$
c_{A}(n)=1+\xi_{1}+\xi_{2}+\ldots+\xi_{n_{0}-1},
$$

где слагаемые независимы и

$$
\mathbf{P}\left(\xi_{i}=1\right)=1-\mathbf{P}\left(\xi_{i}=0\right)=p_{\theta i}, \quad i=1, \ldots, n_{0}-1 .
$$

Отметим, что аналогичное разложение на сумму независимых бернуллиевских слагаемых общего числа циклов случайной подстановки в модели Эвенса было получено в [3], поэтому результаты этой работы могут быть непосредственно переформулированы для рассматриваемого нами случая (это будет сделано позже).

Замечание 2. Для изучения числа $c_{\bar{A}}(n)$ циклов нечетной длины в (18) надо положить $\theta_{1}=0, \theta_{2}=\theta>0$ (напомним, что $d=2$ ), что дает следующий результат:

$$
H_{n A}(\theta)=n !\left[z^{n}\right]\left(\frac{1+z}{1-z}\right)^{\theta / 2}=\sum_{k=0}^{n}\left(\begin{array}{l}
n \\
k
\end{array}\right)\left(\frac{\theta}{2}\right)_{k}\left[\frac{\theta}{2}\right]_{n-k} .
$$

Отсюда видно, насколько сложнее задача изучения распределения числа циклов случайной $\bar{A}$-подстановки (имеющей лишь циклы нечетной длины).

\section{5. $A(r)$-циклы}

Пусть подмножество $A$ имеет вид

$$
A(r)=\{I: i \leqslant r\}, \quad r \geqslant 2
$$

(длины циклов ограничены числом $r$ ). Для этого случая формула (6) принимает вид

$$
\begin{aligned}
H_{n A(r)}(\theta) & =n !\left[z^{n}\right](1-z)^{-\theta_{2}} \exp \left\{\left(\theta_{1}-\theta_{2}\right) \sum_{i=1}^{r} \frac{z^{i}}{i}\right\} \\
& =n !\left[z^{n}\right](1-z)^{-\theta_{1}} \exp \left\{\left(\theta_{2}-\theta_{1}\right) \sum_{i>r} \frac{z^{i}}{i}\right\} .
\end{aligned}
$$


Отметим, что при $r=2$ этот результат совпадает с (14) в случае $d=2$.

Итак, изучение совместного распределения чисел $A(r)$-циклов $c_{A(r)}(n)=\sum_{i \leqslant r} c_{i}$ и $\bar{A}(r)$-циклов $c_{\bar{A}(r)}(n)=\sum_{i>r} c_{i}$ случайной $n$-подстановки в модели (5) может быть основано на исследовании совместной производящей функции (7) этих характеристик с $H_{n A(r)}(\theta)$, указанным в (24).

Рассмотренные примеры, на наш взгляд, убедительно демонстрируют как достаточную универсальность предложенной методики, так и присущие ей сложности: за исключением отдельных случаев (типа (21)-(22)), точные решения в обсуждаемой проблематике имеют форму громоздких комбинаторных выражений, из которых проблематично извлечь конкретную информацию (хотя бы, например, вычислить средние и дисперсии величин $c_{A}(n)$ и $\left.c_{\bar{A}}(n)\right)$.

Поэтому дальнейшее продвижение в этой тематике можно осуществить лишь на пути асимптотического анализа, предполагая что степень подстановки стремится к бесконечности, как это обычно делается в теории случайных подстановок в классической (равновероятной) модели.

Детальный асимптотический анализ предложенной модели будет проведен в следующей работе, а здесь в завершение мы анонсируем один из соответствующих результатов.

Применение метода перевала для случая $d$-конгруэнтных циклов (см. параграф 4) показывает, что при $n \rightarrow \infty$ случайные величины $c_{A}(n)$ и $c_{\bar{A}}(n)$ асимптотически независимы и асимптотически нормальны с параметрами, соответственно, $\left(\left(\left(\theta_{1} / d\right) \ln n,\left(\theta_{1} / d\right) \ln n\right)\right.$, $\left(\left((d-1) \theta_{2} / d\right) \ln n,\left(\left((d-1) \theta_{2}\right) / d\right) \ln n\right)$. В частности, отсюда следует, что общее число циклов $c(n)=\sum_{j=1}^{n} c_{j}(n)$ в такой двухпараметрической модели асимптотически нормально со средним и дисперсией, асимптотически эквивалентными $\left(\left(\theta_{1}+(d-1) \theta_{2}\right) / d\right) \ln n$.

Замечание 3. В заключение отметим, что изложенная методика допускает следующее многомерное обобщение. Пусть задано разбиение множества $X_{n}=\{1,2, \ldots, n\}$

$$
X_{n}=\bigcup_{j=1}^{N} A_{j}, \quad A_{i} \cap A_{j}=\varnothing, \quad i \neq j, \quad n \geqslant 2,
$$

и $c_{A_{j}}(n)=\sum_{i \in A_{j}} c_{i}$ есть число $A_{j}$-циклов для произвольной подстановки $s \in S_{n}$, $j=1,2, \ldots, N$.

Далее, введем на множестве $S_{n}$ вероятностную меру вида

$$
\mathbf{P}_{\theta}(s)=I\left(\sum_{i=1}^{n} i c_{i}=n\right) \prod_{j=1}^{N} \theta_{j}^{c_{A_{j}}} / H_{n}(\theta), \quad \theta=\left(\theta_{1}, \ldots, \theta_{N}\right), \quad \theta \geqslant 0,
$$

где теперь

$$
H_{n}(\theta)=n !\left[z^{n}\right] \exp \left\{\sum_{j=1}^{N} \theta_{j} \sum_{i \in A_{j}} \frac{z^{i}}{i}\right\}
$$

Тогда для совместной производящей функции вектора $\left(c_{a_{j}}(n), j=1, \ldots, N\right)$ справедливо представление (аналог (7))

$$
\mathbf{E}_{\theta} \prod_{j=1}^{N} t_{i}^{c^{A_{j}(n)}}=H_{n}(t \times \theta) / H_{n}(\theta), \quad t \times \theta=\left(t_{1} \theta_{1}, \ldots, t_{N} \theta_{N}\right) .
$$


Такая конструкция позволяет, в принципе, изучать и такие ситуации, когда какието $A$-циклы в подстановке запрещены (в (25)-(26) надо соответствующие параметры $\theta_{j}$ положить равными нулю), а нам важна информация не просто о числе остальных циклов, но и об их более детальной структуризации.

\section{Список литературы}

1. Ивченко Г. И., Медведев Ю. И., Случайные комбинаторные объекты. Доклады РАН (2004) 396, №2, 151-154.

2. Ивченко Г. И., Медведев Ю. И., Случайные подстановки: общая параметрическая модель. Дискретная математика (2006) 18, №4, 105-112.

3. Ивченко Г. И., Медведев Ю. И., О случайных подстановках. Труды по дискретной математике (2002) 5, 73-92.

4. Ewens W. I., The sampling theory of selectively neutral alleles. Theor. Popul. Biol. (1972) 3, 87-112.

5. Сачков В. Н., Введение в комбинаторные методы дискретной математики. МЦНМО, Москва, 2004.

6. Якымив А. Л., Вероятностные приложения тауберовых теорем. Физматлит, Москва, 2005.

7. Якымив А. Л., Предельная теорема для логарифма порядка случайной $A$-подстановки. Дискретная математика (2010) 22, №1, 126-149.

Статья поступила 6.04.2011. 